\title{
Biological Information Processing: Bits of Progress
}

\author{
Nicholas C. Spitzer; Terrence J. Sejnowski
}

Science, New Series, Vol. 277, No. 5329 (Aug. 22, 1997), 1060-1061.

Stable URL:

http://links.jstor.org/sici?sici=0036-8075\%2819970822\%293\%3A277\%3A5329\%3C1060\%3ABIPBOP\%3E2.0.CO\%3B2-D

Science is currently published by American Association for the Advancement of Science.

Your use of the JSTOR archive indicates your acceptance of JSTOR's Terms and Conditions of Use, available at http://www.jstor.org/about/terms.html. JSTOR's Terms and Conditions of Use provides, in part, that unless you have obtained prior permission, you may not download an entire issue of a journal or multiple copies of articles, and you may use content in the JSTOR archive only for your personal, non-commercial use.

Please contact the publisher regarding any further use of this work. Publisher contact information may be obtained at http://www.jstor.org/journals/aaas.html.

Each copy of any part of a JSTOR transmission must contain the same copyright notice that appears on the screen or printed page of such transmission.

JSTOR is an independent not-for-profit organization dedicated to creating and preserving a digital archive of scholarly journals. For more information regarding JSTOR, please contact jstor-info@ jstor.org. 
fluctuations in the detected emission signal, which can be analyzed to gain knowledge about the underlying physical mechanism. In the work of Vanden Bout et al., additional flickering effects (fluorescence amplitude jumps) have been observed for single conjugated polymer molecules (2), a recent entry into the single-molecule arena. In a similar vein, blinking and switching effects have also been reported for single molecules of a novel fluorescent protein (6).

As an example of what "blinking" means, the figure shows a sequence of 100 microscopic images of the emission from a single protein molecule. Its emission blinks on and off even though the pumping laser beam is continuously present. Such fluctuations are now becoming near-universal features of the single-molecule regime, and they provide unprecedented insight into behavior that is normally hidden by the usual ensemble averaging.

Conjugated polymers are a relatively new class of semiconducting materials that combine the electronic and optical properties of semiconductors and the processability of conventional polymers (7). The conjugation results from overlap of the $\pi$-electron orbitals along the chain of the polymer. In the work of Vanden Bout et al. (2), a derivatized poly ( $p$-phenylene vinylene) (PPV)-poly ( $p$ pyridylene vinylene) $(\mathrm{PPy})$ copolymer was chosen, because the photoluminescence and electroluminescence of this and related conjugated polymers show particular promise for light-emitting device (LED) and laser applications. In such systems, one of the more important elementary excitations is an exciton (bound electron-hole pair), which is typically delocalized over a few monomer units and produces an emitted photon when recombination occurs. Such single excitons are known to migrate in semiconductor materials until a recombination center is reached, and several years ago, these single luminescent entities were first observed in a $\mathrm{GaAs} / \mathrm{AlGaAs}$ quantum-well material with a sophisticated scanning near-field optical microscopy technique at liquid-helium temperatures (8).

For the work on the PPV-PPyV copolymer at room temperature, a scanning confocal microscope allowed observation of localized emission spots arising from photogenerated excitons (2). The occurrence of localized spots showed that strong communication occurs along the polymer chain. For each bright spot, a complex flickering (fluctuations in the fluorescence intensity), which was different from spot to spot, was observed, along with eventual photobleaching. Interestingly, by using a clever two-wavelength pumping technique, the workers were able to prove that the flickering was not the result of spectral diffusion effects but rather the generation of a quenching defect at some location along the polymer chain. This conclusion should have important ramifications in the development of these materials for LED and sensor applications. In particular, it will be most important to reduce the concentration of quenching defects in order to maintain high-efficiency light emission for future LED applications.

A key feature of these studies on both single conjugated polymer molecules and on single fluorescent proteins is the observation of novel mechanisms for flickering, blinking, and switching in single quantum emitters. Such blinking and flickering effects would be almost unobservable and certainly misinterpreted in studies on large ensembles, because the various emitters contributing to the overall sum are generally uncorrelated: The mean value of the total emission would simply be smaller. These observations are clear examples of the power of single-molecule spectroscopy and microscopy in opening up a frontier of previously hidden physical effects.

\section{References}

1. Th Basche W E Moerner M Orrit U P. Wild, Eds., Single Molecule Optical Detection, Imaging, and Spectroscopy (Wiley-VCH, Munich 1997); Special Issue on Single Molecules and Atoms, Acc. Chem. Res. 29 (December 1996).

2. D. A Vanden Bout et al., Science 277, 1074 (1997)

3. Analogy attributed to J. S. Skinner (private communication).

4. W. P. Ambrose and W. E. Moerner, Nature 349 225 (1991)

5. J. K. Trautman, J. J. Macklin, L. E. Brus, E. Betzig ibid. 369, 40 (1994); W. P. Ambrose, P. M Goodwin, J. C. Martin, R. A. Keller, Science 265 364 (1994).

6. R. M. Dickson, A. B. Cubitt, R. Y. Tsien, W. E Moerner, Nature 388, 355 (1997)

7. T. A. Skotheim, R. L. Elsenbaumer, J. R Reynolds, Eds., Handbook of Conducting Poly mers (Dekker, New York, 1986); H. G. Keiss, Ed. Conjugated Conducting Polymers (Springer, Berlin, 1992)

8. H. F. Hess, E. Betzig, T. D. Harris, L. N. Pfeiffer, K W. West, Science 264, 1740 (1994).

\title{
Biological Information Processing: Bits of Progress
}

\author{
Nicholas C. Spitzer and Terrence J. Sejnowski
}

Are there principles of information processing common to all biological systems, whether simple or complex, fast or slow? A recent conference (1) that brought together researchers from a wide range of disciplines hinted that there are. Several key principles of biological information processing emerged, and new computational methods were presented for analyzing complex biological systems. There were two prominent themes of the meeting.

The first was the many ways in which biochemical reactions within cells can be used for computation. A variety of biological processes-concatenations of chemical amplifiers and switches-can perform computations such as exponentiation, differentiation, and integration. These computational cascades include metabolic processes as diverse as gene transcription, cell cycle timing, and decoding oscillations of second messengers (2). Enzymatic amplification, regulatory

N. C. Spitzer is in the Department of Biology and Center for Molecular Genetics, University of California at San Diego, La Jolla, CA 92093, USA. E-mail: nick@biomail.ucsd.edu. T. J. Sejnowski is at the Howard Hughes Medical Institute, Salk Institute for Biological Studies, La Jolla, CA 92037 and Department of Biology, University of California at San Diego, La Jolla, CA 92093, USA. E-mail: terry@salk.edu

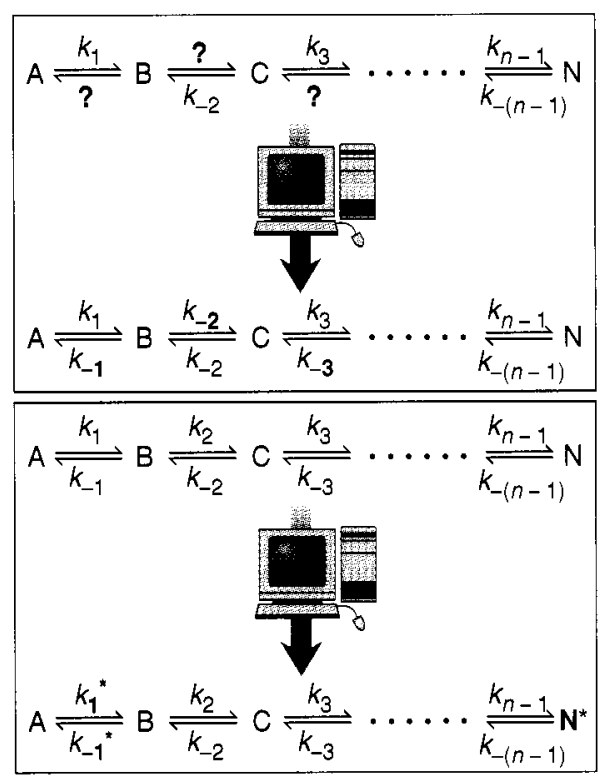

Fig. 1. Computing by chemical switches. (Top) Simulation of signaling or metabolic cascades allows assignment of unidentified rate constants for specific steps in the pathway generating the product N. (Bottom) Optimizing the cascade to achieve a different outcome $\left(N^{*}\right)$, which mimics an evolutionary process, identifies critical rate constants that change and focuses further investigation on specific components 
feedback, and parallel pathways lead to chemical networks with dynamical properties comparable to those found in both biological and artificial neural networks, but on time scales that range over eight orders of magnitude, from milliseconds to days. In some systems, the response properties of the chemical reactions filter the information so that the same chemical messenger can carry several signals simultaneously on different time scales (3).

Biological systems for which the full cascade is known can be modeled by computer-a form of digital reconstitution. A computer simulation of a biochemical cascade is a summary of the current understanding of the system and can be used to predict the outcomes of perturbations. These models provide insights into the ways in which behaviors and cellular responses are modulated or stabilized and focus experimental investigation on specific molecules.

One of the best-studied behaviors based on a chemical network is bacterial chemotaxis, in which a cascade of chemical switches leads to phosphorylation of protein $Y$ and activation of the flagellar motor. Simulation of the molecular interactions generating bacterial chemotaxis allows determination of rate constants for different reactions (see Fig. 1, top) and shows how activities of particular chemotactic proteins are affected by their allosteric interactions. Deviations from observed bacterial behaviors hint at undetected components or unsuspected interactions in the natural signaling pathway. The effects of overexpression or knockout of components, mimicking processes that produce polymorphisms, can also be assessed $(4,5)$ (see Fig. 1, bottom). Systematic variation of parameters identifies relatively invariant, and thus robust, features of the signaling cascade (6).

The embryonic development of Drosophila, in which a series of distinct segments defined by stripes of expression of transcription factors are elaborated, entails molecular signaling by similar cascades. A connectionist model of development reveals the dynamical interactions among transcription factors through equations that describe the spatiotemporal patterns previously determined experimentally ( 7$)$.

Although the idea that living organisms operate by chemical switches may be intuitively simple, the resulting dynamics can lead to surprising behaviors. Bacteria climb chemical gradients, but exhibit a decreased response to increased levels of chemoattractant receptor $(4,5)$. Simulating expression of stripes of the evenskipped (eve) gene in Drosophila requires relatively large diffusivity of regulatory Kruppel protein and small diffusivity of eve protein, providing a theoretical basis for eve's observed anatomical localization (7). In modeling prokaryotic protein production, construction of a statistical model and simulation of gene expression suggest that alternative regulatory pathways may be selected probabilistically, consistent with phenotypic variability in isogenic populations (8).

The second theme was pulses as carriers of information-many biological systems generate pulsatile activity, achieving superior signal-to-noise ratios by frequency modula-

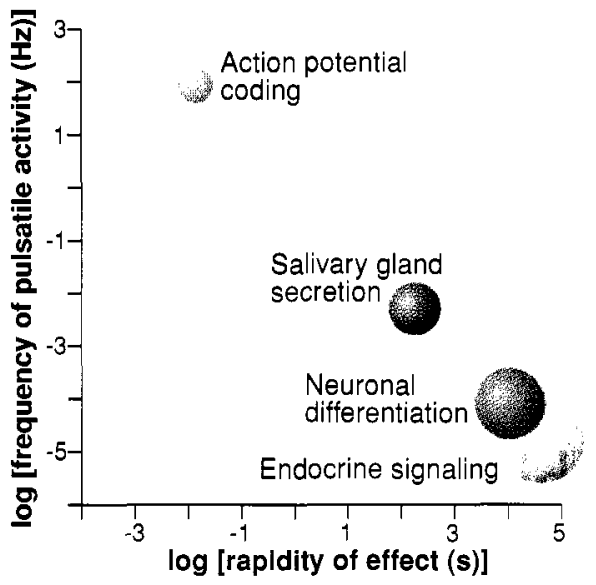

Fig. 2. When timing is everything. The frequency of signaling is proportional to the rapidity (time) of the effect on a log-log scale, across many orders of magnitude.

tion (FM) coding and avoiding desensitization to sustained signals (by multiple mechanisms). For example, insect salivary gland secretion depends on the frequency of transient increases of intracellular calcium, on a time scale of minutes (9). Differentiation of amphibian spinal neurons requires patterns of calcium transients at particular frequencies, on a time scale of hours (10). In these neurons, the appearance of a neurotransmitter, GABA ( $\gamma$-aminobutyric acid), and maturation of potassium current require a low frequency of calcium transients, whereas axon extension requires a higher frequency, and neurons are tuned to differentiate normally in response to the frequencies of calcium transients they generate. Secretion of growth hormone is pulsatile on a time scale ranging from minutes to days, a property that is clearly important because pulsatile application yields greater weight gain and bone growth than continuous application (11). The frequency of coding is proportional to the rapidity of the readout of the signals (see Fig. 2).

Unlike most engineered communications systems that use well-defined frequencies to transmit information, many biological signaling systems use fluctuating signals. Counterintuitively, in several systems stochastic phenomena can enhance the precision of the outcomes. Genetic circuitryfrom clonal variation in bacterial chemotaxis to gene transcription in eukaryotes-relies on random association of molecules with targets to ensure specific results on a time scale of minutes. Another biological system in which random patterns may be used to achieve highly precise outputs is the cerebral cortex, where the timing of neuronal action potentials is regular but unreliable in response to repeated, constant inputs. However, the spike-initiating mechanism responds with reliability on a time scale of milliseconds in response to repetition of a fluctuating input (12) like the fluctuating membrane potentials of neurons in vivo. These results raise the possibility that the timing of spikes carries information in addition to that carried by the average firing rate. Introduction of sufficiently wide bandwith noise amplifies the signal generated by voltage-dependent ion channels (13) and may contribute to action potential timing.

Traditional disciplines often become highly productive when they intersect with other disciplines at critical times in their independent evolution. This conferencewhich brought together chemists, physicists, information theorists, developmental biologists, endocrinologists, and neuroscientists-bore some resemblance to the seminal convergence of physicists, chemists, and biologists at the 9th Cold Spring Harbor Symposium on Quantitative Biology, on genes and chromosomes, in 1941. In the pre-DNA era this group forged a recognition that general principles cut across all species from plants to humans. The time is now ripe to search for general mechanisms supporting the dynamic kaleidoscopic dance of biological information processing that surrounds the static information contained in the genome.

\section{References and Notes}

1. International Conference on Biological Information Processing, Hanover, Germany, 2 to 4 March 1997; organized by $G$. Brabant and K. Prank. Sponsored by Deutsche Forschungsgemeinschaft and Ferring Arzneimittel GmbH.

2. A. Arkin and J. Ross, Biophys, J. 67, 560 (1994)

3. M. J. Berridge, Nature 386, 759 (1997)

4. D. Bray, R. B. Bourret, M. I. Simon, Mol. Biol. Cell 4, 469 (1993).

5. D. Bray, Nature 376,307 (1995)

6. N. Barkai and S, Leibler, ibid. 387, 913 (1997); L. Hartwell, ibid., 0. 855.

7. J. Reinitz and D. H. Sharp, Mech. Dev. 49, 133 (1995)

8. H. H. McAdams and A. Arkin, Proc. Natl. Acad. Sci. U.S.A. 94, 814 (1997)

9. M. J. Berridge and P. E. Rapp, J. Exp. Biol 81, $217(1979)$

10. X. Gu and N. C. Spitzer. Nature 375, 784 (1995).

11. K. Prank, M. Kloppstech, S. J. Nowlan, T. J. Sejnowski, G. Brabant, Biophys. J. 70, 2540 (1996).

12. Z. F. Mainen and T. J. Sejnowski, Science $\mathbf{2 6 8 ,}$ 1503 (1995); F, Rieke, D. Warland, R, van Steveninck. W. Bialek, Spikes: Exploring the Neural Code (MIT Press, Cambridge, MA, 1997)

13. S. M. Bezrukov and I. Vodyanoy, Nature 385, 319 (1997). 